University of Nebraska - Lincoln

DigitalCommons@University of Nebraska - Lincoln

Faculty Publications in Computer \& Electronics Electrical \& Computer Engineering, Department Engineering (to 2015)

2012

\title{
LTE PHY Performance Analysis under 3GPP Standards Parameters
}

\author{
Fahimeh Rezaei \\ University of Nebraska-Lincoln, frezaei@unlnotes.unl.edu \\ Michael Hempel \\ University of Nebraska-Lincoln, mhempel2@unl.edu \\ Hamid Sharif \\ University of Nebraska-Lincoln, hsharif@unl.edu
}

Follow this and additional works at: https://digitalcommons.unl.edu/computerelectronicfacpub

Part of the Computer Engineering Commons

Rezaei, Fahimeh; Hempel, Michael; and Sharif, Hamid, "LTE PHY Performance Analysis under 3GPP Standards Parameters" (2012). Faculty Publications in Computer \& Electronics Engineering (to 2015). 82. https://digitalcommons.unl.edu/computerelectronicfacpub/82

This Article is brought to you for free and open access by the Electrical \& Computer Engineering, Department of at DigitalCommons@University of Nebraska - Lincoln. It has been accepted for inclusion in Faculty Publications in Computer \& Electronics Engineering (to 2015) by an authorized administrator of DigitalCommons@University of Nebraska - Lincoln. 


\title{
LTE PHY Performance Analysis under 3GPP Standards Parameters
}

\author{
Fahimeh Rezaei, Michael Hempel, Hamid Sharif \\ Department of Computer and Electronics Engineering \\ University of Nebraska-Lincoln, Omaha, NE, USA \\ \{frezaei, mhempel, hsharif\}@unlnotes.unl.edu
}

\begin{abstract}
Long Term Evolution (LTE) has been introduced by 3GPP (3rd Generation Partnership Project) and is poised to dominate the 4th generation (4G) of mobile telecommunication networks. In this article, we present an in-depth analysis of the LTE physical layer's characteristics and its performance. Our work is unique in providing a detailed performance study based on Release 8 of the 3GPP standard. Several works have discussed the LTE performance; however, most have been restricted to limited scenarios. Our work shows a more comprehensive investigation of the maximum data throughput under different conditions and scenarios. Our performance study includes TDD and FDD operational modes for uplink and downlink transmissions in different antenna diversity schemes, data modulation, and code rates. Our results show that LTE (3GPPRelease 8) supports downlink throughputs of up to 300Mbps and for the uplink a throughput of up to $75 \mathrm{Mbps}$.
\end{abstract}

Keywords-LTE; Performance; 3GPP standard; PHY; FDD; TDD; OFDM; SC-FDMA; frame layout

\section{INTRODUCTION}

3GPP specified LTE [1-7], also called E-UTRA (EvolvedUniversal Terrestrial Radio Access), to promote HSPA (High Speed Packet Access) performance through reducing overhead. LTE Downlink modulation is based on OFDM (Orthogonal Frequency Division Multiplex) which provides multi user access, robustness to time dispersion of radio channel, and low complexity for receiver design. SC-FDMA (Single Carrier Frequency Division Multiple Access) is employed in uplink that provides low peak-to-average power ratio.

LTE supports both FDD (Frequency Division Duplex) and TDD (Time Division Duplex) schemes, resulting in different frame structures in the time domain. In the frequency domain, different numbers of Resource Blocks (RBs) can be addressed based on the system bandwidth, which varies from $1.4 \mathrm{MHz}$ to $20 \mathrm{MHz}$. A single RB consists of a group of carriers for transferring data. Each RB is $0.5 \mathrm{~ms}$ in time domain and 180 $\mathrm{kHz}$ in frequency domain. Depending on the cyclic prefix mode and subcarrier spacing, the number of subcarriers and OFDM symbols in downlink (SC-FDMA symbols in Uplink) is different for each RB [1].

The rest of the paper presents a literature review in section II, the E-UTRA architecture overview in section III, and a general description of the LTE physical layer in section IV. In section V our LTE performance results are presented, with our conclusions shown in section VI.

\section{LITERATURE REVIEW}

Different characteristics and features of LTE have been presented in the literature. In [8], an overview of LTE specifications is provided and its performance is verified by simulation results. Comprehensive description of the link layer protocols and the interaction between protocol layers is discussed in [9]. Physical layer characteristics and features are investigated in [10] and the results show how different features such as adaptive modulation, scheduling, and multi antenna transmission schemes impact the spectral efficiency. However, the throughput analysis is limited to SISO scenarios. Another important consideration is the performance comparison of WiMAX and LTE based on different scenarios. In [11], the presented results illustrate that LTE is better than WiMAX with respect to spectrum efficiency, average user throughput, and cell edge bit rate gains for both TDD and FDD operations. This study also shows the results for $10 \mathrm{MHz}$ system bandwidth. In [12] the performance assessment is limited to downlink and FDD operation only and the results present better radio performance for LTE because of the lower overhead. In addition to performance comparisons, analogous specifications and technologies are collated in different publications. For instance, the work in [13] classifies MIMO configurations in LTE and WiMAX, while [14] describes relay technologies for both communication standards. However, the large variations in the reported performance results prompted us to take a more in-depth analysis of the LTE specifications and develop a comprehensive and detailed evaluation of the LTE performance.

In this study, we model and simulate an LTE link with exclusion of all the overhead subcarrier allocations of control and broadcast channels as well as reference signals. We also considered $20 \mathrm{MHz}$ system bandwidth in order to analyze the maximum throughput possible for different scenarios. Moreover, our performance study includes TDD and FDD operational modes in different antenna diversity schemes, data modulation, and code rates. The results of our study are presented in this paper.

\section{E-UTRA ARCHITECTURE}

The next generation of 3GPP network architecture is known as LTE, whose air interface is referred to as E-UTRAN. Complementing E-UTRAN in LTE is the System Architecture Evolution (SAE) of the core network that defines EPC (Evolved Packet Core). EPC and EUTRAN together comprise the Evolved Packet System (EPS) that is shown in figure 1. EPC includes the Packet Data Network Gateway (P-GW), 
Serving Gateway (S-GW), Mobility Management Entity (MME), Home Subscriber Service (HSS), Policy Control and Charging Rules Function (PCRF)[3].

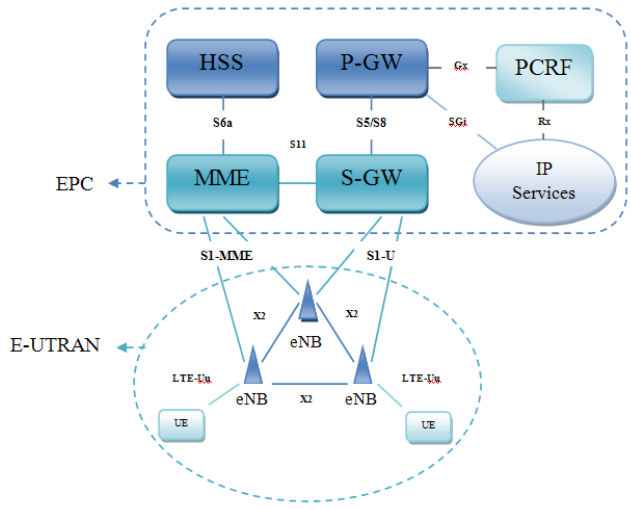

Fig. 1. EPS Architecture [15]

P-GW operates as an IP anchor and connects UE to the external packet data network. P-GW filters the downlink user IP packets and performs policy enforcements. User IP packets are transferred via S-GW that is also operating as a local mobility anchor when UE moves between eNBs. While P-GW can be considered as a mobility anchor between 3GPP and non3GPP technologies, S-GW operates as mobility anchor between other 3GPP technologies. MME is responsible for functionalities such as user authentication, bearer management, and ideal mode UE tracking UE and core network by NonAccess Stratum (NAS) protocol. The information on the Packet Data Networks (PDNs) to which the UE can connect is located in HSS. PCRF manages charging functionalities and policy control as well as authorizing QoS.

\begin{tabular}{|cc|} 
Table 1. List of PHY Channels & \\
\hline PHY Channel Name & Acronym \\
\hline Physical Broadcast Channel & PBCH \\
\hline Physical Multicast Channel & PMCH \\
\hline Physical Downlink Shared Channel & PDSCH \\
\hline Physical Uplink Shared Channel & PUSCH \\
\hline Physical Downlink Control Channel & PDCCH \\
\hline Physical Random Access Channel & PRACH \\
\hline Physical Uplink Control Channel & PUCCH \\
\hline
\end{tabular}

LTE radio access consists of four protocol layers: PHY, MAC, Radio Link Control (RLC), and Packet Data Convergence Protocol (PDCP). PHY and MAC utilize transport blocks to exchange information. At the PHY layer, several channels are allocated for data and control communication as specified in table 1 .

\section{LTE PHY DESCRIPTION}

LTE physical layer supports multiple users in downlink using OFDM (Orthogonal Frequency Division Multiplexing) and multiple access in uplink using SC-FDMA (Single CarrierFrequency Division Multiple Access). However, whereas OFDM is a multi-carrier scheme, SC-FDMA is a single-carrier channel access scheme that provides the advantage of a low Peak-to-Average-Power Ratio (PAPR). LTE operates either using FDD (Frequency Division Duplexing) or TDD (Time Division Duplexing).

Radio frames for downlink and uplink transmission are $10 \mathrm{~ms}$ long. In TDD LTE uses two $5 \mathrm{~ms}$ half-frames. Half frames are further divided into 4 subframes and one special subframe, based on the downlink-to-uplink switch point periodicity. TDD frame structure can thus be configured into 7 different formats of $1 \mathrm{~ms}$-subframes. In FDD, each frame simply consists of ten $1 \mathrm{~ms}$-subframes. Each subframe in both FDD and TDD has two slots of $0.5 \mathrm{~ms}$ each. In LTE, PHY resource allocation is organized in units of resource blocks (RB). Each resource block is a rectangular structure containing resource elements (RE), or subcarriers, of the same modulation type. The most common configuration is 7 symbols by 12 subcarriers per RB. A list of all possible RB configurations is shown table 2 .

\begin{tabular}{|c|c|c|c|}
\hline & \multirow{2}{*}{$\begin{array}{c}\text { Normal Cyclic Prefix } \\
\text { Subcarrier } \\
\text { Spacing: } 15 \mathrm{kHz}\end{array}$} & \multicolumn{2}{|c|}{ Extended Cyclic Prefix } \\
\hline & & $\begin{array}{c}\text { Subcarrier } \\
\text { Spacing: } 15 \mathrm{kHz}\end{array}$ & $\begin{array}{c}\text { Subcarrier } \\
\text { Spacing: } 7.5 \mathrm{kHz}\end{array}$ \\
\hline DL & $7 \times 12$ & $6 \times 12$ & $3 \times 24$ \\
\hline UL & $7 \times 12$ & $6 \times 12$ & - \\
\hline
\end{tabular}

Each of the two slots per subframe is 1 resource block wide in time domain and contains between 6 and 110 resource blocks for $1.4 \mathrm{MHz}$ to $20 \mathrm{MHz}$ channel bandwidth, respectively, in frequency domain.

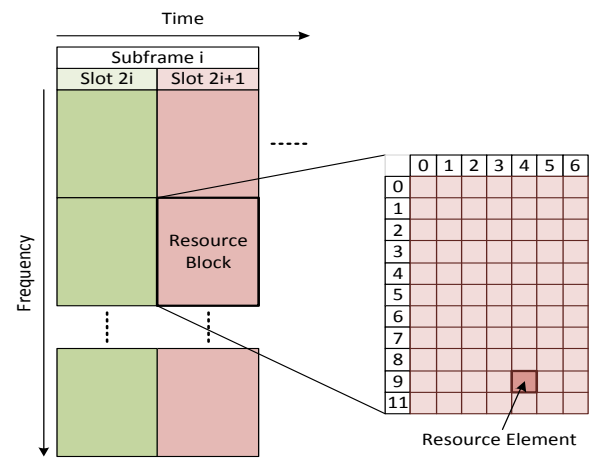

Fig. 2. Resource Block Structure

All of the different PHY channels of LTE, as well as reference signals, are then mapped onto this structure of resource elements. An example for DL mapping is shown in figure 3 . Figure 4 illustrates the UL mapping scheme.

In tables 3, 4 and 5 we have summarized the allocation counts for resource elements per $10 \mathrm{~ms}$-frame to the different channels for FDD-DL, FDD-UL, and TDD for different antenna configurations and channel bandwidths. For these calculations, we assumed that 1 (for $20 \mathrm{MHz}$ bandwidth) or 2 (for $1.4 \mathrm{MHz}$ Bandwidth) OFDM symbols are used for PDCCH. The number of resource elements allocated for PDCCH and PDSCH changes proportionally based on the number of OFDM symbols assigned for PDCCH $(1,2,3$ or 4 OFDM symbols can be occupied by PDCCH). In uplink transmissions, $\mathrm{PUCCH}$ format mode $2,2 \mathrm{a}$, or $2 \mathrm{~b}$ is assumed $[1]$. 


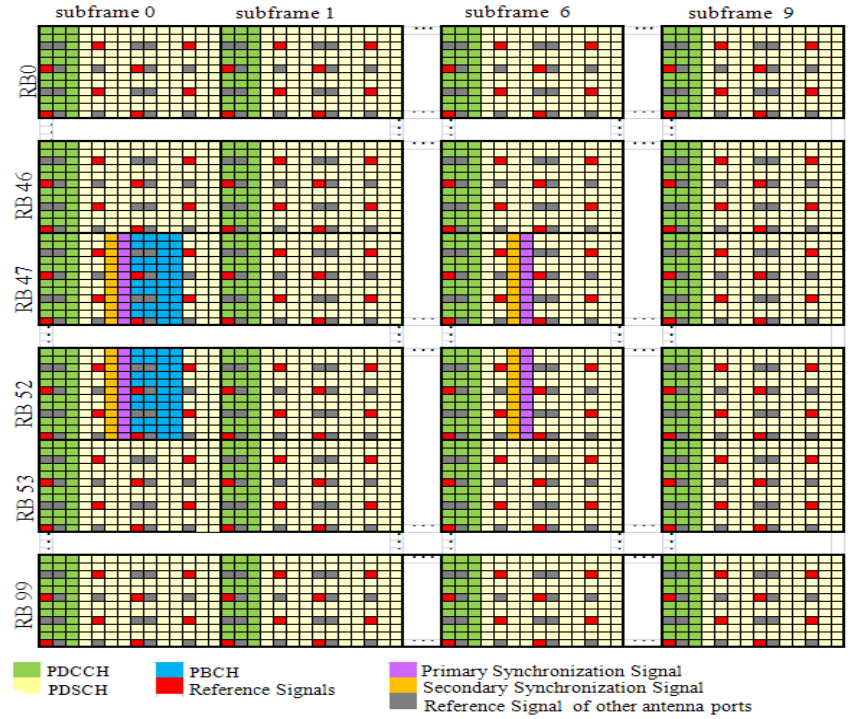

Fig. 3. DL Configuration for $20 \mathrm{MHz}$ FDD with $15 \mathrm{kHz}$, Normal CP

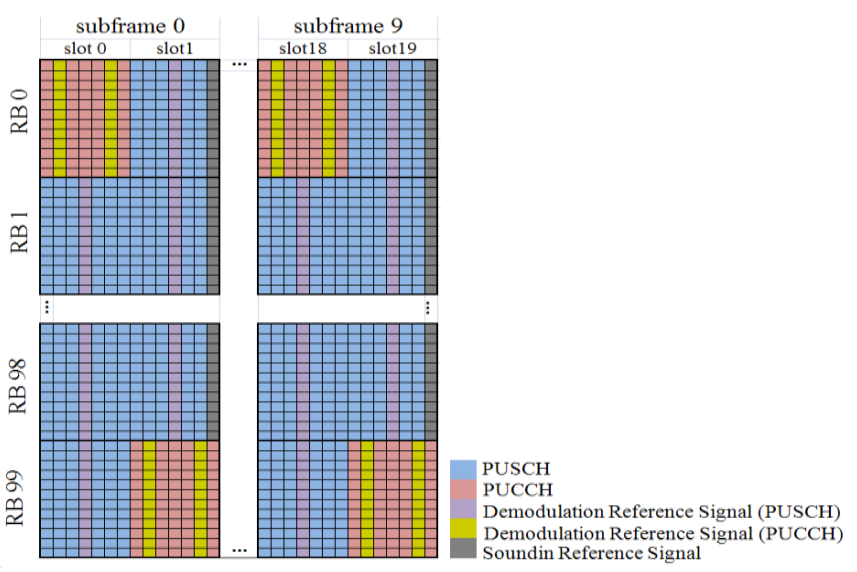

Fig. 4. UL Configuration for $20 \mathrm{MHz}$ FDD with $15 \mathrm{kHz}$, Normal CP

The allocation of PUCCH differs by using 1,1a, or $1 \mathrm{~b}$ configuration [1]. Although two antenna ports can exist in uplink, only one antenna is used for data transmissions at a time, resulting in the values of table 4 . In table 5, the resource element calculation in TDD mode is based on a single antenna port. Therefore, these tables only represent a subset of possible layouts.

\section{Performance Results}

For this study we have performed a comprehensive analysis of all frame layout configurations to determine the performance characteristics of LTE. Our results are shown and discussed in this section. We provide the performance analysis results of both LTE downlink and uplink physical layer for different scenarios of FDD operation in section A and TDD operation in Section B. Our results are based on MATLAB simulations for which the relevant parameters are summarized in table 6 . For better understanding of the LTE Performance boundaries, results are classified as two main categories: $1.4 \mathrm{MHz}$ of system bandwidth utilizing SISO, and the maximum antenna diversity mode supported with $20 \mathrm{MHz}$ in downlink and uplink.
Table 3. FDD-DL Resource Allocation

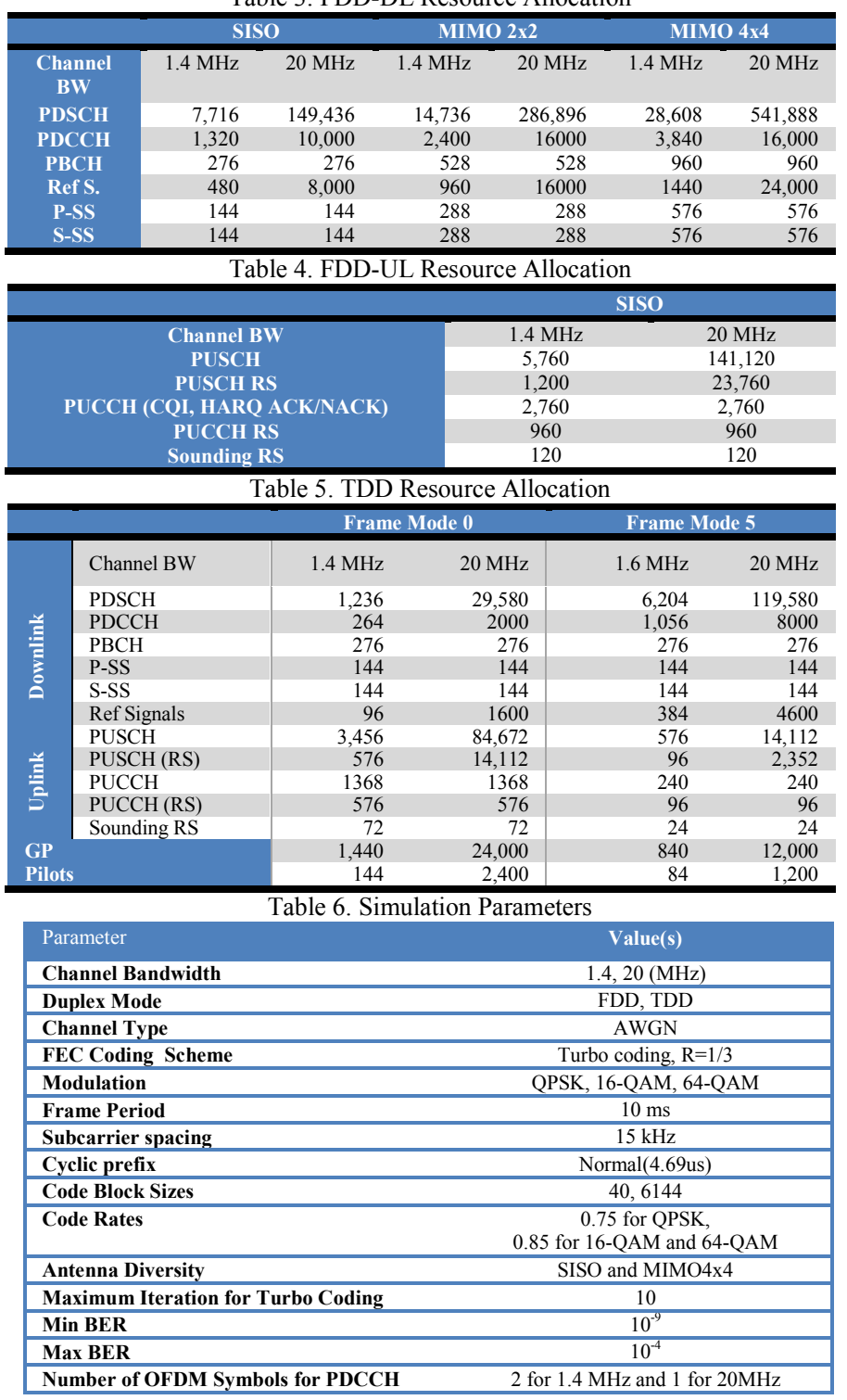

Figure 5 illustrates the bit error rate (BER) values for the minimum and maximum codeword sizes using QPSK, 16QAM and 64-QAM. As shown in this figure, since the turbo coder performs better with longer code block sizes, the SNR gain required for a 6144-bit block size is $4 \mathrm{~dB}$ less than the one required for 40-bit code blocks. The throughput calculations are then carried out using the resulting frame error rate (FER) values for the corresponding minimum and maximum codeword sizes. The presented figures demonstrate the throughput values for the PDSCH and PUSCH user data channels.

\section{A. LTE FDD Throughput and Performance}

In this section, our LTE throughput simulation results are discussed for FDD operation based on mentioned parameters in table 6. As presented in figure 6, when the eNB uses the minimum bandwidth for transferring data to the user equipment, the maximum throughput of $3.93 \mathrm{Mbps}$ in the PDSCH channel can be achieved by using 64-QAM and a code 
rate of 0.85 in SISO. As we can recognize in this figure, reaching the full throughput for 40-bit code blocks requires about $8 \mathrm{~dB}$ of SNR gain, while using 6144-bit blocks requires only $4 \mathrm{~dB}$ gain. When operating with $20 \mathrm{MHz}$ system bandwidth, $4 \times 4$ MIMO and 64-QAM with a code rate of 0.85 , 276.36 Mbps of data throughput can be achieved as demonstrated in figure7. Comparing the results in figure 7 to the results of figure 6 we can observe that the resource blocks used for the transmission of PDSCH in figure 7 is almost 16 times that of the one when using $1.4 \mathrm{MHz}$ in figure 6. Also, the number of antennas for data transmission is increased to 4 . By using the maximum supported code rate of 0.92 , the maximum throughput can be improved to $299.12 \mathrm{Mbps}$.

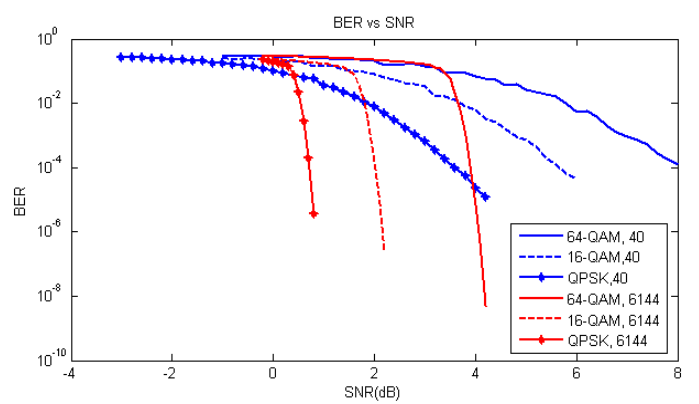

Fig. 6. BER vs SNR for 40 and 6144 code words

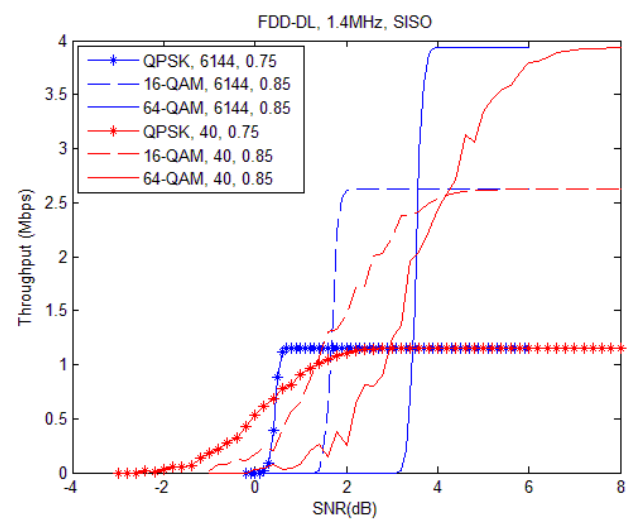

Fig. 8. FDD Downlink Throughput in 1.4 MHz Bandwidth and single antenna layer

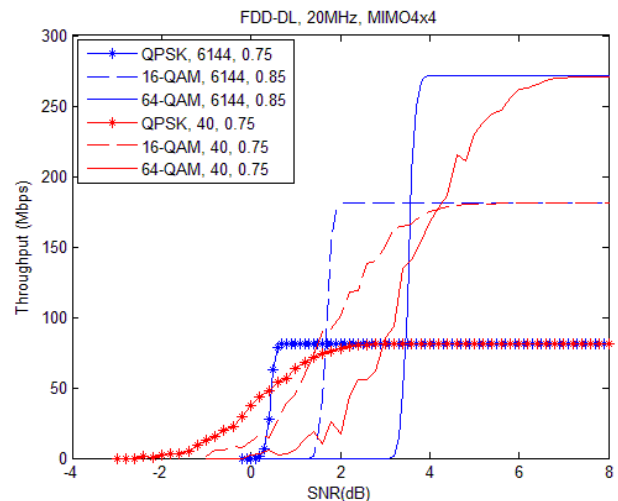

Fig. 9. FDD Downlink Throughput in $20 \mathrm{MHz}$ bandwidth and four antenna layers

Figures 8 and 9 present the maximum PUSCH throughput in $1.4 \mathrm{MHz}$ and $20 \mathrm{MHz}$ bandwidth, respectively. In figure 8, which uses $1.4 \mathrm{MHz}$ of bandwidth, the UE can send up to 2.93 Mbps of data when 64-QAM and code rate 0.85 is considered. However, in a $20 \mathrm{MHz}$ bandwidth system the uplink provides $71.97 \mathrm{Mbps}$ of throughput in the same scenario. Generally we can observe that in the uplink, due to the fact that by increasing the bandwidth (resource blocks), the absolute overhead of control channels approximately remains the same, the capacity grows by almost 17 times, which is proportional to the bandwidth expansion.

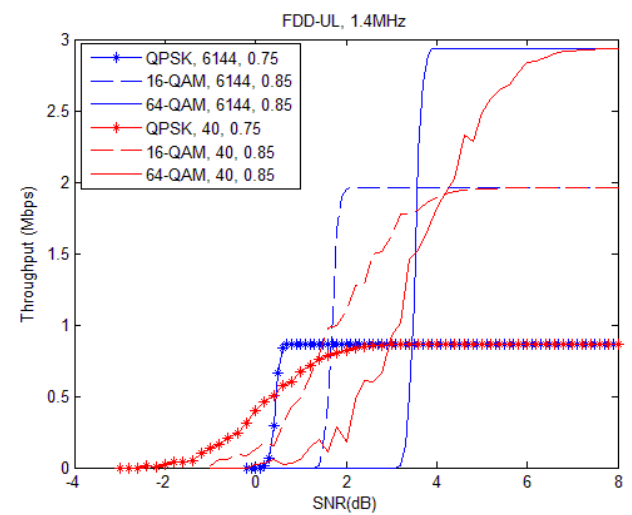

Fig. 10. FDD Uplink Throughput in $1.4 \mathrm{MHz}$ Bandwidth

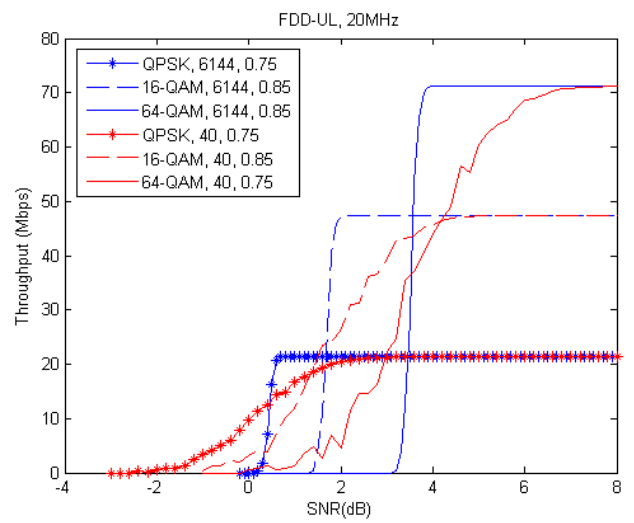

Fig. 11. FDD Uplink Throughput in $20 \mathrm{MHz}$ Bandwidth

\section{A. LTE TDD Throughput and Performance}

In figures 10 and 11, we presented the PDSCH and PUSCH throughput for two different modes of TDD (Mode0 labeled as M0 and Mode5 labeled as M5 in the figures)[1]. The first structure for special subframe is chosen where 3 OFDM symbols is assigned for DwPTS, 1 for UpPTS[1]. The reason for choosing these two modes out of others is due to the fact that, compared to the other 7 modes, in Mode 0 the maximum number of subframes (6 subframes) are assigned for uplink transmission. Similarly in Mode 5, downlink occupies the most subframes possible ( 8 subframes) for data transmissions. The maximum throughput calculation is based on a $20 \mathrm{MHz}$ channel bandwidth using, 64-QAM, and 0.85 puncture code rate. In mode 0 , due to the fact that eNB uses 2 subframes for data transmission to the UE, PDSCH conveys up to 54.70 Mbps of information. However, in mode 5, which carries downlink information in 8 subframes, the throughput improves to $221.16 \mathrm{Mbps}$. Employing the maximum code rate improves the downlink throughput to $239.38 \mathrm{Mbps}$. PUSCH occupies 6 
subframes for data transmissions in Mode 0, providing 43.18 Mbps as the maximum UL throughput. In Mode5 using one subframe for UL transmissions, only 7.19 Mbps of throughput is feasible.

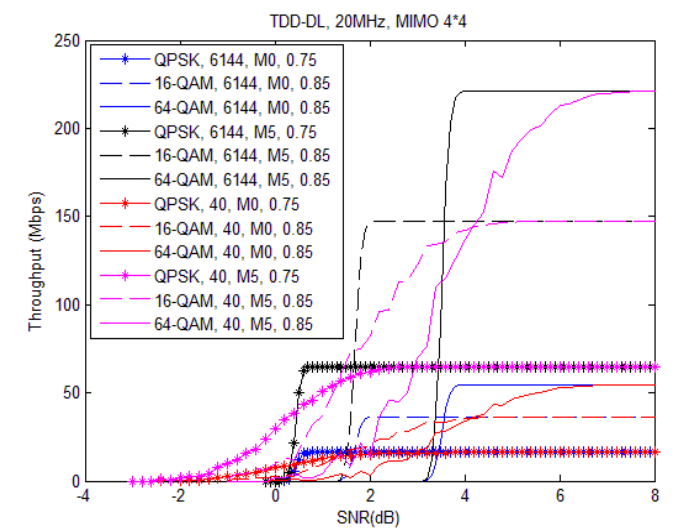

Fig. 12. TDD Downlink Throughput in 20MHz Mode0 and Mode5 for four antenna layers

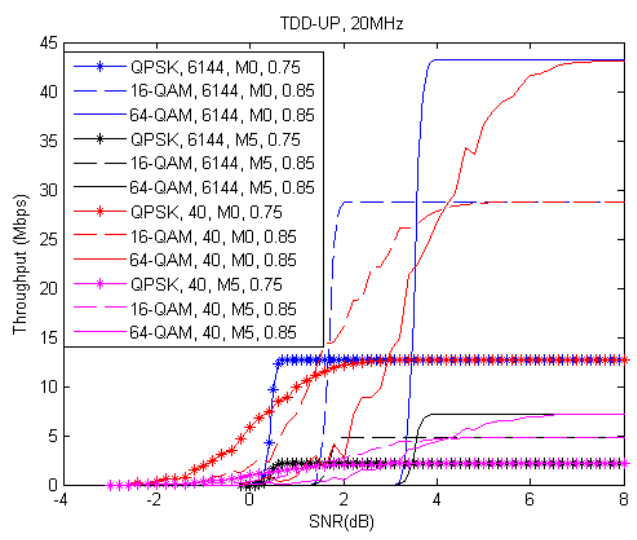

Fig. 13. TDD Uplink Throughput in $20 \mathrm{MHz}$ Mode0 and Mode5

\section{SUMMARY}

In response to the existing lack of an in-depth and comprehensive LTE performance study, we presented a detailed analysis of the LTE physical layer in this paper and discussed the performance of LTE under different scenarios. Our main objective was to provide a comprehensive study of the maximum uplink and downlink throughput for the 3GPP standard Release8 in both FDD and TDD operations under different configurations and scenarios. We have also presented our results for the minimum and maximum system bandwidth for better understanding of LTE throughput boundaries. Our performance study includes the TDD and FDD operational modes in different antenna diversity schemes, data modulation, and code rates.

Our study is based on Release 8, which is currently being deployed. 3GPP Release 8 is followed by Release9 with minor enhancements to the air interface. Release10, also known as LTE-Advanced, is still under development to fulfill the requirements for IMT-Advanced, such as $1 \mathrm{Gbps}$ of data rate in low mobility. We will investigate the performance impact of these new releases as they are finalized.
The presented results in this paper show that LTE can achieve 276.36 Mbps of throughput for PDSCH when using a 0.85 puncture code rate and assuming 1 OFDM symbol assigned for PDCCH. However, by using a 0.92 puncture code rate, the maximum downlink data throughput improves to 299.12 Mbps. The maximum data throughput for PUSCH is $71.97 \mathrm{Mbps}$ for 2 antenna layers using 64-QAM and a 0.85 puncture code rate. In TDD operation, the maximum PDSCH throughput is $239.38 \mathrm{Mbps}$ and $43.18 \mathrm{Mbps}$ for PUSCH.

\section{ACKNOWLEGEMENT}

This study was funded and conducted by the Advanced Telecommunications Engineering Laboratory (www.TEL.unl.edu) at the University of Nebraska-Lincoln.

\section{REFERENCES}

[1]. 3GPP TS 36.211, Evolved Universal Terrestrial Radio Access (EUTRA), "Physical Channels and Modulation", (Release 8).

[2]. 3GPP TS 36.213, Evolved Universal Terrestrial Radio Access (EUTRA), "User Equipment (UE) Radio Transmission and Reception", (Release 8).

[3]. 3GPP TS 36.212, Evolved Universal Terrestrial Radio Access (EUTRA), "Modulation and channel coding", (Release 8).

[4]. 3GPP TS 36.300, Evolved Universal Terrestrial Radio Access (EUTRA), "Overall description", (Release 8).

[5]. 3GPP TS 36.201, Evolved Universal Terrestrial Radio Access (EUTRA), “Long Term Evolution, General description”, (Release 8).

[6]. 3GPP TS 36.101, Evolved Universal Terrestrial Radio Access (EUTRA), "User Equipment (UE) radio transmission and reception, (Release 8).

[7]. 3GPP TS 36.104, Evolved Universal Terrestrial Radio Access (EUTRA) "Base Station (BS) radio transmission and reception", (Release 8).

[8]. D. Astély, E. Dahlman, A. Furuskär, Y. Jading, M. Lindström, S. Parkvall, "LTE: the evolution of mobile broadband", IEEE Communication Magazine, April, 2009.

[9]. A. Larmo, M. Lindström, M. Meyer, G. Pelletier, J. Torsner, H. Wiemann, "The LTE link-layer design", IEEE Communication Magazine, April, 2009.

[10]. J J. Sánchez, D. Morales-Jiménez, G. Gómez, J. T. Enbrambasaguas, "Physical Layer Performance of Long Term Evolution Cellular Technology", IST Mobile and Wireless Communications Summit, IEEE Conference, July, 2007.

[11]. A. Furuskär, T. Jönsson, and M. Lundevall, "The LTE Radio Interfacekey Characteristics and Performance" PIMRC IEEE Conference, September, 2008.

[12]. C. Ball, T. Hindelang, I. Kambourov, S. Eder, "Spectral Efficiency Assessment and Radio Performance Comparison between LTE and WiMAX”, PIMRC IEEE Conference, September 2008.

[13]. J. Lee, J-k. Han, J. Zhang, "MIMO Technologies in 3GPP LTE and LTE-Advanced", EURASIP journal on Wireless Communications and Networking, May, 2009.

[14]. Y. Yang, H. Hu, J. Xu, G. Mao, "Relay Technologies for WiMAX and LTE-Advanced Mobile Systems", IEEE Communication Magazine, October, 2009.

[15]. 3GPP TS 33.401, Technical Specification Group Services and System Aspects; "Architecture enhancements for non-3GPP accesses", (Release 8). 